\title{
Acquired Pigmentation of Porcine Lymph Nodes: Dietary Polyphenolic Compounds as Biological Markers?
}

\author{
Walter Pinna ${ }^{1}$, Maria Grazia Cappai ${ }^{1}$, Ilaria Sogos ${ }^{2}$, Giuseppa Nieddu ${ }^{2}$, Maurizio Picciau ${ }^{2}$ \\ ${ }^{1}$ Department of Agraria, University of Sassari, Sassari, Italy; ${ }^{2}$ Ex-Faculty of Veterinary Medicine, University of Sassari, Sassari, Italy. \\ Email: mgcappai@uniss.it
}

Received October $24^{\text {th }}, 2012$; revised November $24^{\text {th }}, 2012$; accepted December $2^{\text {nd }}, 2012$

\begin{abstract}
The investigation aimed at exploring whether 1) high contents of natural polyphenols from the diet can induce pigment accumulation in lymph nodes (LNs); 2) if so, whether polyphenolic compounds and derivates can be used as biological markers; 3 ) and whether a lymph node from a specific anatomical region can be univocally identified, so as to be considered as a sentinel for the identification of the dietary origin of pigments. A paired match approach was used to switch 20 pigs (range of initial body weight, BW: $113-121 \mathrm{~kg}$ ) to two experimental diets, for four weeks: ten pigs (pair housed) were fed with an experimental acorn based diet (acorns: $50 \%$ in the diet, as fed; total polyphenols, $78.1 \mathrm{~g}$ $\mathrm{TP} / \mathrm{Kg} \mathrm{DM}$ in the diet; tannic acid equivalent, $25.8 \mathrm{~g} \mathrm{TAE} / \mathrm{kg} \mathrm{DM}$ ); the remainder ten, received a pelleted complete diet for finishers ( $0 \%$ acorns in the diet). Daily feed intake in the last two weeks of the experimental feeding was recorded per pair of pigs in both groups of animals, showing an average intake of $610 \mathrm{mg} \mathrm{TAE} / \mathrm{kg} \mathrm{BW} / \mathrm{d}$. At an average final BW of between 127 to $137 \mathrm{~kg}$, all pigs were slaughtered and LNs from different anatomical regions of the carcass were removed and analysed. At gross inspection, LNs from both groups displayed different grades of intensity and diffusion of pigmentation: a partial and incidental pigmentation was randomly detected in renal or sub-iliac LNs in the control group; a constant and uniform pigmentation of LNs was observed in acorns fed pigs: a dark brown staining diffused to the whole LN associated with a brownish colour of the muscles was found systematically. At light microscope intracytoplasmic granules were found in macrophages and dendritic cells from both groups, but, at confocal laser analysis, an intense auto-fluorescence was observed in medial-iliac LNs from the carcasses of acorn-fed pigs (green emission). However, intracellular sources of blue and green fluorescence at different wavelengths, likely due to tryptophan, indoleamine and derivates were also found in medial-iliac and inguinal LNs from the control group. A dietary origin was attributed to the different discoloration of LNs between the carcasses of the two groups: such acquired pigmentation is relevant in the left sub-iliac LN, but the confocal laser microscopic test to elicit auto-fluorescence of polyphenolic compounds (biological markers) displayed a $76.9 \%$ specificity, despite a $100 \%$ of sensitivity for the univocal identification of the carcass from acorn-fed pigs. Cranial sternal LNs resulted to suit the sentinel role in the distinction of carcass from acorns fed pigs at confocal laser microscopic analysis.
\end{abstract}

Keywords: Acorns; Auto-Fluorescence; Lymph Node; Marker; Total Polyphenols

\section{Introduction}

\subsection{Acorns as Dietary Source of Natural Polyphenols}

In different Mediterranean Countries, typical lard-type hog productions are often based on free ranged pig farming [1-6]. That way, pigs can directly consume ripe acorns (oak fruits) from the ground or into mixed meal diets: in the latter case, acorns are collected by the breeder and administered to ranged finishers, in combinations with other raw feedstuffs. The shredded acorn proportion consists of about the $50 \%$ of the mixed meal, together with cereals and legume seeds. When pigs are put into land use, acorns represent the main energy source among homegrown feedstuffs, during fall and winter [7-13]. According to the chemical composition of acorns, starch results in between of the $51 \%$ to $57 \%$ of dry matter and crude fat content ranges between the $4 \%$ to $6 \%$ (DM basis). The practice of feeding hulled acorns ensures stability in quality and preserved hygienic conditions of the seed and prolonged storage time, on one side, and an economic advantage on the other, as de-hulling would be expensive for the breeder. Nevertheless, ripe acorns display also to possess high amounts of anti-nutritional compounds (tannins as plant secondary metabolites, within polyphenols), which pigs showed to tolerate successfully $[9,14]$, in con- 
trast to other animal species [15-18].

\subsection{Lymph Node Pigmentation}

In the literature, the carcass of acorn-fed pigs are reported to display a macroscopic pigmentation of lymph nodes but a normal colour of other tissues, such as fat, spleen, fat, liver and bone marrow [19]. More recently, the acquired melanosis of LNs in the carcass from free ranged pigs was also described and a dietary origin was appointed to be the cause [20], likely due to acorn consumption. However, no feeding trials were carried out to clearly assess the dietary origin of pigments and the descriptions re- ported in the literature appear to be controversial to date $[19,20]$. As a consequence, no information about the effects of dietary polyphenolic compounds and dosages on the induced lymph node pigmentation from acorns consumption are reported.

\subsection{Auto-Fluorescence of Natural Polyphenols}

An elicited auto-fluorescence of natural polyphenols was recently described. In particular fisetin and myricetin are described to possess fluorogenic properties [21] and appear to belong to the list of flavonoids of oak acorns [22]. The fluorogenic properties of natural polyphenols might be deployed to set a rapid, cheap and reliable method to elicit the autofluorescence in mammal tissues, at confocal laser microscopic analysis on fresh samples, without the use of external stain.

This investigation was undertaken with the purpose of determining: 1) whether an experimental acorn based diet ( $50 \%$ of acorn combined in the diet, as fed) high in polyphenolic compounds is effectively capable of inducing lymph node pigmentation in comparison with a complete $\operatorname{diet}(0 \%$ of acorn in the diet); 2$)$ if the pigmentation of lymph nodes occurs, whether lymph nodes from a distinctive anatomical region can be identified to behave as a sentinel; 3) and whether polyphenolic compounds and derivates can be used as biological markers to distinguish the traditional carcasses from acorn-fed pigs.

\section{Materials and Methods}

\subsection{Animals and Diets}

Animal handling followed the recommendations of European Union directive 86/609/EEC and Italian law 116/92 concerning animal care.

A total of 20 cross-bred (Duroc sires on Large White $\mathrm{X}$ German Landrace) finishing pigs (body weight range at start, BW: 113 to $121 \mathrm{~kg}$ ) were involved for the feeding trial. Pigs were divided into two experimental groups, according to a pair match approach: after an adaptation period of 10 days when all animals were fed the same pelleted complete diet (phase 1), ten pigs were switched to the experimental diet with increasing proportions of hulled shredded acorns $(10 \%, 20 \%, 30 \%, 40 \%$, phase 2$)$ until reaching the $50 \%$ of acorn combined in the diet as fed (phase 3), in a mixed meal based on cereals (wheat, $35 \%$ of the diet as fed) and legume seeds (peas and faba beans, $15 \%$ of the diet, as fed); the rest of pigs continued to be fed with the pelleted complete diet. The chemical composition of the two experimental diets is reported in Table 1.

Daily feed intake per each pair of pigs in both groups was recorded: each left over was removed from the feeder and weighed, from the previous day. On the basis of weight and chemical composition of feed and left over, determinations were used to calculate DM, TP and TAE intakes per $\mathrm{kg} \mathrm{BW}$.

The chemical composition of diet and feed left over was carried out according to Weende analysis, and modified methods [23], in duplicates. The total polyphenols and the tannin acid equivalent were determined by FolinCiocalteau (1927) modified method [24]. All animals were sacrificed eight $\mathrm{h}$ after the last meal (on day 29).

\subsection{Investigations on Lymph Nodes}

The pigs were slaughtered in a same abattoir, following the same phases and procedures across the slaughtering chain. The lymph nodes (LNs) of the carcasses from pigs of both dietary treatments were explored, at the end of the slaughtering chain, at abattoir. The LNs from the different anatomical regions were considered for the in situ inspection:

- Mandibular, cranial sternal, aortic thoracic, renal, aortic lumbar, medial iliac, sacral, and superficial inguinal LNs;

- At dissection, other LNs from further different districts of the carcass were removed for the inspection and included:

Table 1. Analyzed chemical composition of the experimental diets.

\begin{tabular}{lcc}
\hline $\begin{array}{l}\text { Acorns [\%] in the diet } \\
\text { Chemical composition of the diets }\end{array}$ & 0 & 50 \\
\hline Dry matter [g/kg, as fed] & 890 & 751 \\
Crude ash [g/kg DM] & 68.5 & 62.3 \\
Crude protein [g/kg DM] & 175 & 116 \\
Crude fibre [g/kg DM] & 45.0 & 95 \\
Starch [g/kg DM] & 390 & 410 \\
Total Polyphenols & $<1.0^{*}$ & 78.1 \\
Tannic acid equivalent [g/kg DM] & $<1.0^{*}$ & 25.8 \\
${ }^{\circ} \mathrm{ME}(\mathrm{MJ} / \mathrm{kg} \mathrm{DM})$ & 13.1 & 10.8 \\
\hline
\end{tabular}

"below the lowest concentration for equipment detection; ${ }^{\circ} \mathrm{ME}$ : Metabolizable Energy (MJ/kg $\mathrm{DM})$, calculated according the formula: $\mathrm{ME}(\mathrm{MJ})=0.0223 \times \mathrm{CP}+0.0341 \times \mathrm{EE}+0.017 \times$ Starch $+0.0168 \times$ Sugars $+0.0074 \times(\mathrm{DM}-\mathrm{Ash}-\mathrm{CP}-\mathrm{EE}-$ Starch - Sugars $-\mathrm{CF})-0.0013 \times$ CF. 
- Parotid, axillary, deep mediastinal, iliofemoral, superficial and deep popliteal LNs.

A total of 260 pairs of $\mathrm{LN}$ were screened out: the gross inspection considered the appearance of the capsule (with particular regard to colour) and the parenchyma at cut. Eventual lymphadenopathy assessment was based on the absence/presence of abscessation, since a lymphadenomegaly is difficult to establish in pigs, due to the naturally occurring different size and shape of LNs from different anatomical regions in the same individual.

The microscopic evaluation of LNs tissue, was carried out both on fresh (130 LNs) and fixed (130 LNs) samples, using the contralateral LN. Fresh LNs were frozen in embedding medium (Tissue-Tek@1988 Miles Inc., IN, 46515 USA) and cut $(2 \mu \mathrm{m})$ with the cryostat. After an incubation in a phosphate buffered saline solution (PBS) for 30 minutes, samples were ready to be observed at a confocal laser fluorescence microscope.

Fixed (neutral formaldehyde 12.5\%) and paraffin embedded samples, were cut $(2 \mu \mathrm{m})$ with the microtome and stained with routinary technique (Haematoxylin-Eosin, HE) for light microscope analysis.

\subsection{Calculations and Statistical Analysis}

Individual feed consumption was averaged on daily feed intake (FI) across the last two weeks of the experimental feeding, expressed in $\mathrm{g}$ of diet, as fed. In addition, the individual consume of feed per day was also related to the respective $\mathrm{BW}$, in each pig from both experimental groups and expressed as:

$$
\mathrm{FI}_{\text {rel }}=\mathrm{g} \times \mathrm{kgBW}^{-1} \mathrm{~d}^{-1}
$$

Individual DM intake (DMI) per day was also related to the respective BW and such value was used to calculate the $\mathrm{g}$ of TAE intake by each pig at an ingestion of $100 \mathrm{~g}$ of DM of the diets.

Body mass development was calculated on the BW gain (four weeks period) expressed as percentage of increase in BW from the beginning of the experimental feeding plan.

Values in the tables are presented as arithmetical means with standard deviation (Mean \pm SD).

The confidence of the dark pigmentation of LNs due to polyphenols from the diet was related to the prevalence $(\mathrm{P}, \%)$ of the pigmented (diffused and uniform dark brown capsule vs spotted and irregular brownish capsule) in the screened LN, calculated in each group of animals as diet-dependent:

$$
\text { Prevalence: } P=\frac{n_{s}^{1}}{\left(n_{s}^{1}+n_{s}^{h}\right)} \times 100
$$

where $\mathrm{n}_{\mathrm{s}}^{1}=$ number of pigmented LN, $\mathrm{n}_{\mathrm{s}}^{\mathrm{h}}=$ number of LN with no or partial pigmentation.

Furthermore, sensitivity (or true positive rate, TPR), specificity (or true negative rate, TNR) and accuracy (ACC) of the elicited fluorescence determined in LNs through confocal laser microscopic test were calculated using the following formulas:

$$
\begin{aligned}
& \mathrm{TPR}=\frac{\mathrm{TP}}{(\mathrm{TP}+\mathrm{FN})} \times 100 ; \\
& \mathrm{TNR}=\frac{\mathrm{TN}}{(\mathrm{FP}+\mathrm{TN})} \times 100 ; \\
& \mathrm{Acc}=\frac{(\mathrm{TP}+\mathrm{TN})}{(\mathrm{TP}+\mathrm{TN}+\mathrm{FP}+\mathrm{FN})} \times 100,
\end{aligned}
$$

where TP is the number of LNs with fluorogenic compounds from pigs fed with the acorn combined diet, $\mathrm{FP}$ is the number of LNs with fluorogenic compounds from pigs fed with the pelleted complete diet, TN is the number of LNs with no fluorogenic compounds in all the pigs, FN is the number of LNs that were apparently not pigmented but with microscopic fluorogenic compounds.

The statistic significance of results between the two experimental groups was evaluated by means of Student's T-test.

\section{Results}

\subsection{Daily Feed Intakes and Dietary Tannins Ingestion from Different Acorns Inclusion in the Experimental Diets}

The daily feed intake in pigs fed with the acorn combined diet was higher than in pigs fed with the control diet, though the daily DMI in relation to $\mathrm{kg}$ of body weight resulted similar (Table 2) between groups. The acorn fed group showed an average daily intake of $610 \mathrm{mg} \mathrm{TAE} / \mathrm{kg}$ $\mathrm{BW} / \mathrm{d}$ in the last two weeks of experimental feeding. The body mass gain was significantly lower $(p<0.05)$ in animals fed with the acorns based diet, due to the different energy concentration (MJ of calculated Metabolizable Energy, ME per kg of dry matter in the diet) of the two diets (Table 1). No selective behaviour could be pointed out in the acorn fed pigs, as non substantial differences could be observed between the chemical composition of the origi-

Table 2. Intake and of feed and polyphenolic compounds in pigs from the two experimental diets.

\begin{tabular}{cccc}
\hline $\begin{array}{c}\text { Acorns [\%] in the diet } \\
\text { Intake of polyphenols }\end{array}$ & 0 & vs & 10 \\
\hline Group [No. of animals] & 10 & vs & $127 \pm 2.80$ \\
BW at slaughter [kg] & $137 \pm 2.10$ & & $23.6 \pm 1.72$ \\
$\begin{array}{c}\text { DM intake } \\
\left.\text { [g } \cdot \mathrm{kg} \mathrm{BW}^{-1} \cdot \mathrm{d}^{-1}\right]\end{array}$ & $26.6 \pm 0.67$ & vs & \\
$\begin{array}{c}\text { Tannins intake } \\
{\left[\mathrm{mg} \cdot \mathrm{kg} \mathrm{BW}^{-1} \cdot \mathrm{d}^{-1}\right]}\end{array}$ & $<1^{*}$ & vs & $610 \pm 0.01$ \\
\hline
\end{tabular}

${ }^{*}$ below the lowest concentration for equipment detection. 
nal acorn based diet and the feed left over. All animals from both groups appeared healthy, despite the tannin rich diet (from acorn combination in the mixed meal) consumption: this is in agreement with previous own results [14].

\subsection{Gross Inspection of LNs}

From the inspection of LNs on the carcass at the abattoir, a dark generalized colour was pointed out in all LNs from the different anatomical regions of the carcass from the totality of acorn fed pigs: in details, the capsule of LNs appeared of a homogeneous and diffused dark brown colour, on the whole surface of the LN (130 LNs out of 130 ). The LNs in the carcass from the pellet fed pigs displayed to possess a randomly partial and incidental pigmentation in renal or sub-iliac LNs: in the latter case, the discoloration of the LNs was limited to parts of the LN and did not involve systematically all other explorable LNs from the same individual (Figure 1).

A longwise cut (symmetrical in respect to the hilum) was carried out in all the LNs from the carcasses of both groups and the parenchyma was explored: no creaks at cut and abscessations could be pointed out. A dark brown stained pathway could be observed in LNs from the acorn-fed pigs, marking the procollagene or stroma (Figure 2): the brownish streams through the lymphatic circulation get deeper into the medulla from the paracortical region (lymphatic sinus), rendering clearly visible the peculiar anatomo-physiology of the lymph node of the pig, which differs from all other domestic mammals species [25] for its organogenesis.

\subsection{Microscopic Features of Lymphnodal Cell Population}

The light microscopic analysis did not allow to point out any (physio)-pathognomonic peculiarity for the distinction between lymph nodes from the two dietary treatments. However, it is to remark that the dark intracytoplasmic granules in the macrophages and in the dendritic cells could be observed in all lymph nodes from both experimental groups ( $50 \%$ vs $0 \%$ acorn in the diet). Therefore, the light microscopic analysis on LNs tissue stained with HE was not sufficient to assess the origin of the pigmentation of LNs in the acorn fed group.

The confocal laser microscopic test was able to elicit the auto-fluorescence of fluorogenic compounds phagocytosed by macrophages and dendritic cells in the LNs, previously screened at gross inspection. All the LNs (130 LNs out of 130) from the acorn fed pigs showed an elicited auto-fluorescence in the range of wavelength of between 450 to $560 \mathrm{~nm}$ (blue and green emissions) independently of the anatomical regions they were removed from: it is to underline that the most diffused fluorogenic

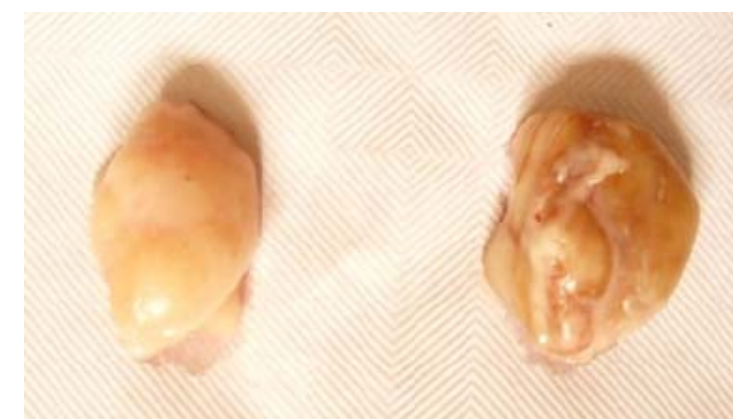

Figure 1. Lymph nodes of pigs from the two experimental groups. Comparison between fresh lymph nodes removed from the carcass of the experimental pigs (left: control group vs right: acorn fed group). At gross inspection, the capsule of the LN appears bright and smooth in both samples. A dark uniform discoloration can be pointed out in the LN on the right, diffused to the whole surface, due to the uptake of dietary polyphenols after the ingestion of the acorn combined diet (78.1 g TP/kg DM).

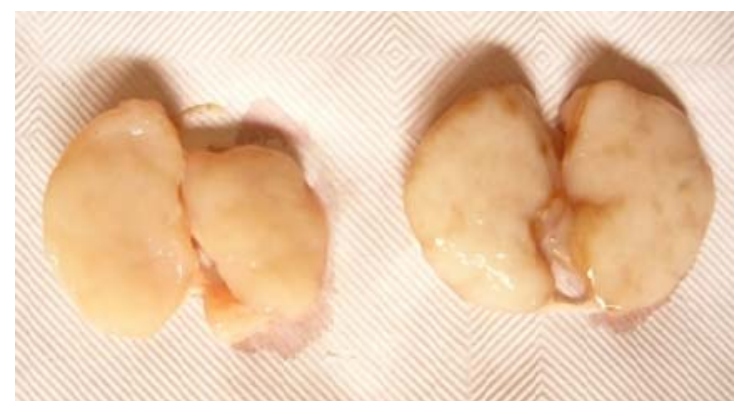

Figure 2. Lymph nodes of pigs from the two experimental groups (parenchyma). A longwise cut through the hilum splits into two halves the same lymph nodes displayed in Figure 1. The brown stream in the right LN describes the organization of the paracortical multiplicity of lymphatic sinus, to the medulla of the organ.

compounds belonged to the medial iliac, sacral and iliofemoral LNs. Such occurrence was also observed in medial iliac, sacral and iliofemoral LNs (30 out of 130) of the control group, though it was not observed in the remainder LNs (2 LNs, only blue emission, wavelength of $387 \mathrm{~nm}$ ) from the other anatomical regions (Figure 3).

A pivotal sentinel $\mathrm{LN}$ can be represented by the cranial sternal LNs: constantly positive at elicitation of fluorescent granules in the acorn fed pigs, appeared negative in the control animals. In support, at gross inspection, LNs from the acorn fed pigs did show a dark brownish overall stain diffused to the whole LN, whereas in the control animals they incidentally appeared to be partly discoloured: the cranial sternal LN is a feasible diagnostic reference $\mathrm{LN}$ as it appears to be easily reachable in the carcass with no additional cuts.

On the basis of the elicited fluorescence and response of LNs, the opportunity offered by the test carried out by the confocal laser microscopic analysis turned out to 


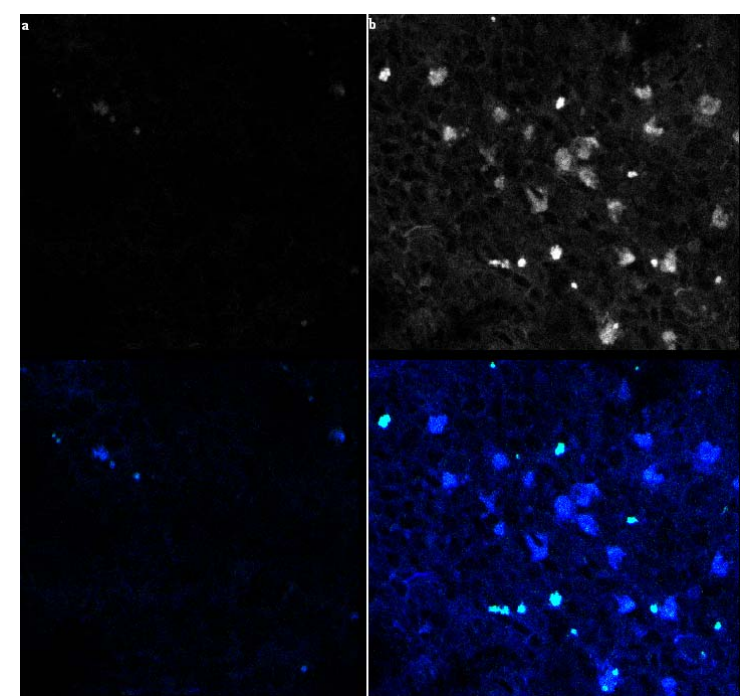

Figure 3. Microscopic picture of Lymph nodes at confocal laser microscope, elicited autofluorescence $(40 \times)$. 3 Elicited auto fluorescence at confocal laser microscopic analysis observed in the $\mathrm{LN}$ from the experimental pigs. It is possible to observe the conspicuous presence of light emitting spots in the $L N$ in the right (b, top frame, black and white; $b$, bottom frame, merged fluorescence in blue and green light, medial-iliac LN, 40×) from the acorn fed group. No light emitting spot can be highlighted on left (a, top frame), except some diffused intrinsic blue light (a, bottom frame) in the medial-iliac $\mathrm{LN}$ from the control group, presumably from endogenous fluorophores (Indoleamin, Trp, NAD(P)H).

have strong sensitivity $(100 \%$, or true positive rate) and good accuracy $(88.5 \%)$, but fair specificity $(76.9 \%$, or true negative rate).

\section{Discussion}

The inspection carried out on LNs removed from viscera and deep anatomical regions of the carcass from the acorn fed pigs highlighted a diffused pigmentation: such finding allowed to distinguish the acorn-fed from the pellet-fed pigs. At this regard, the LNs from the acorn fed pigs showed a constant pattern, which differed substantially from the appearance of the LNs from the control group, which did not display a constantly uniform and diffused discoloration. This is in agreement with previous reports [19], and might be useful to the hypothesis described to be an acquired pseudomelanosis, where a dietary aetiology from the consumption of acorns by free ranged pigs [20] was supposed: actually, a partial staining can be pointed out also in pigs which did not consume acorns in the diet, though with different characteristics.

The literature reports a correlation between the pres- ence of intracytoplasmic granules in lymphatic cells and acorn consumption by the pig [19]: our results highlight that this occurrence is not confined to acorn consumption solely. Nevertheless, as Carta (1948) pointed out, a different origin can be attributed to the granules detectable in the macrophages and dendritic cells of swine LNs, but this implies further specific histochemical investigations, in order to assess which metabolic way leads to the accumulation of endogenous pigment (melanins, lipofuscines, lipochromes, hemosiderin, hematoidin).

Fisetin and myricetin are polyphenols contained in acorns [22] and have been listed within the natural fluorophores (substances displaying fluorogenic properties after elicitation) [21]. Due to the diffused fluorescence (phagocytosed granules) observed in the totality of LNs from the carcasses of the acorn fed pigs, the effective role of dietary polyphenols can be correlated to the presence of external pigments in a systemic way, via lymphatic circulation. As a matter of fact, an overall brownish colour characterized also the muscles of the carcass from the acorn fed group. A different explanation is given to the autofluorescence of some positive LNs from the control group: in the last decade, cellular sources of intrinsic emissions were identified in live tissues [26], and valuable diagnostic perspectives were correlated. NAD $(\mathrm{P}) \mathrm{H}$, flavins, retinol, and tryptophan (Trp) and its indoleamine derivatives were listed among fluorogenic compounds. A major source of intracellular blue emission is $\mathrm{NAD}(\mathrm{P}) \mathrm{H}_{-}$ $\mathrm{NAD}(\mathrm{P})_{\text {, }}$, which is fluorescent only when reduced. Nicotinamide adenine dinucleotide (NA-DH) fluorescence has long been used as an indicator of cellular metabolic state. Distinct UV and blue emission patterns of microvilli in the small intestine of mouse were also described [26]. The occurrence of blue emissions and bright spots in medialiliac, sacral and iliofemoral LNs shows that they possibly indicate indoleamine storage: a same description was given by Zipfel et al. (2003) [26]. That way, the presence of indoleamin and derivates storage should be a common occurrence in those LNs which drain from lymphatic vessels of the caecum and of large bowel of the pig: in the chyme content, Trp and derivates are in large part metabolized by the resident flora. The occasional and inconstant findings of blue emitting granules in LNs from other districts of the control carcasses can be explained by the reduction of $\mathrm{NAD}(\mathrm{P})$ and somewhat to a possible increased local immune system stimulation, and therefore, independent from the dietary origin of pigments.

Indeed, the investigation on the auto-fluorescence of polyphenols in LNs was always reliable when addressing the carcasses from acorn fed pigs; the calculated fair sensitivity highlights the fact that due to the presence of other intrinsic fluorophores in the totality of LNs studied, elicited fluorescence from indoleamin or Trp or NAD(P) 
$\mathrm{H}$ might bias the results: the test allowed to correctly identify the $76.9 \%$ of LNs from the control group (true negative). Thus, further analyses should be carried out to exclude false positive LNs. The acorn combined diet significantly determined the peculiar pigmentation of LNs in a constant way. The peculiarity of the discoloration was here described for the first time as an effect of the experimental feeding based on raw shredded acorns in the diet (providing high amounts of polyphenolic compounds): this allowed to distinguish between the acquired pigmentation of LNs after acorns consumption and the incidental and partial pigmentation of LNs of the pigs, we found though no acorns in the diet were included. The possibility to use the elicited auto-fluorescence of polyphenols without external stain of the tissues represents a rapid and cheap method: it is an immediate test on fresh samples and has no costs of reagents to check the presence of polyphenols. Our results underline that particular care must be paid when assessing lymph nodes features for diagnostic purposes, since the role of biologically active compounds of dietary origin (polyphenols) must be taken into account. The strong sensitivity and fair specificity of the test carried out by the confocal laser microscopic analysis is a pivotal method that aims at improving the laboratory techniques to identify the presence of dietary polyphenol accumulation in the body.

\section{REFERENCES}

[1] C. J. López-Bote, "Sustained Utilization of the Iberian Pig Breed," Meat Science, Vol. 49, No. S1, 1998, pp. S17-S27.

[2] B. Chiofalo, L. Liotta, A. Zumbo and L. Chiofalo, "Seasonal Variations of Free Fatty Acids in Plasma of "Nero Siciliano' Pigs Living in Extensive Conditions," Veterinary Research Communications, Vol. 27, No. 1, 2003, pp. 253- 255. doi:10.1023/B:VERC.0000014153.61928.ec

[3] A. Zumbo, B. Chiofalo, D. Piccolo and L. Chiofalo, "Pigs Reared Outdoor and Plein Air," Journal of Animal Science, Vol. 2, 2003, pp. 379-381.

[4] L. Liotta, B. Chiofalo, A. Zumbo and V. Chiofalo, "Effects of Different Nutritional Levels on Nero Siciliano Pig Performance," Journal of Animal Science, Vol. 4, No. 2S, 2005, pp. 470-472.

[5] S. Porcu, E. Daga, S. Pintus, M. G. Usai, R. Comunian and S. Ligios, "The Sarda Breed Pig: History, Management and Perspectives," 2007.

www.rivistadiagraria.org

[6] A. Olivares, A. Daza, A. I. Rey and C. J. López-Bote, "Effect of Diet Saturation on Growth Performance, Carcass Characteristics and Fat Quality of Heavy Pigs," Food Science and Technology International, Vol. 16, 2010, pp. 312-327.

[7] R. Nieto, M. Rivera, M. A. Garca and J. F. Aguilera, "Amino Acid Availability and Energy Value of Acorn in the Iberian Pig," Livestock Production Science, Vol. 77,
No. 2, 2002, pp. 227-239. doi:10.1016/S0301-6226(02)00040-4

[8] W. Pinna, G. Nieddu, G. Moniello and M. G. Cappai, "Vegetable and Animal Food Sorts Found in the Gastric Content of Sardinian Wild Boars (Sus scrofa meridianalis)," Journal of Animal Physiology and Animal Nutrition, Vol. 91, No. 5-6, 2007, pp. 252-255. doi:10.1111/j.1439-0396.2007.00700.x

[9] M. G. Cappai, P. Wolf, V. Grosse-Liesner, A. Kastner, G. Nieddu, W. Pinna and J. Kamphues, "Effect of Whole Acorns (Quercus pubescens) Shred Based Diet on Parotid Gland in Growing Pigs in Relation to Tannins," Livestock Science, Vol. 134, No. 1, 2010, pp. 183-186. doi:10.1016/j.livsci.2010.06.136

[10] V. Rodríguez-Estévez, A. García and A. G. Gómez-Castro, "Characteristics of the Acorns Selected by Free Range Iberian Pigs during the Montanera Season," Livestock Science, Vol. 122, No. 2, 2009, pp. 169-176. doi:10.1016/j.livsci.2008.08.010

[11] V. Rodríguez-Estévez, A. García and A. G. Gómez-Castro, "Intrinsic Factors of Acorns that Influence the Efficiency of Their Consumption by Iberian Pigs," Livestock Science, Vol. 122, No. 2, 2009, pp. 281-285. doi:10.1016/j.livsci.2008.09.011

[12] V. Rodríguez-Estévez, M. Sánchez-Rodríguez, A. García and A. G. Gómez-Castro, "Feed Conversion Rate and Estimated Energy Balance of Free Grazing Iberian Pigs," Livestock Science, Vol. 132, No. 1-3, 2010, pp. 152-156. doi:10.1016/i.livsci.2010.05.019

[13] D. Tejerina, S. García-Torres, M. Cabeza de Vaca, F. M. Vázquez and R. Cava, "Acorns (Quercus rotundifolia Lam.) and Grass as Natural Sources of Antioxidants and Fatty Acids in the 'Montanera' Feeding of Iberian Pig: Intra- and Inter-Annual Variations," Food Chemistry, Vol. 124, No. 3, 2011, pp. 997-1004. doi:10.1016/j.foodchem.2010.07.058

[14] M. G. Cappai, P. Wolf, W. Pinna and J. Kamphues, "Dose-Response Relationship between Dietary Polyphenols from Acorns and Parotid Gland Hypertrophy in Pigs," Food and Nutrition Sciences, Vol. 3, No. 9, 2012, pp. 1261-1268. doi:10.4236/fns.2012.39166

[15] W. Kaufmann, H. Hagemeister and G. Dirksen, “Adaptation to Changes in Dietary Composition, Level and Frequency of Feeding," In: Y. Ruckebush and P. Thivend, Eds., Digestive Physiology and Metabolism in Ruminants, Avi Publishing Company, Inc., 1980, pp. 587-602. doi:10.1007/978-94-011-8067-2 28

[16] J. A. Neser, J. A. Coetzer, J. Boomker and H. Cable, "Oak (Quercus robur) Poisoning in Cattle," Journal of the South African Veterinary Association, Vol. 53, No. 3, 1982, pp. 151-155.

[17] C. A. Cummings, K. J. Kopedge and A. W. Confer, "Equine Gastric Impaction, Ulceration and Perforation Due to Persimmon (Dyospiros virginiana) Ingestion," Journal of Veterinary Diagnostic Investigation, Vol. 9, No. 3, 1997, pp. 311-313. doi: $10.1177 / 104063879700900315$

[18] T. Shimada and T. Saioth, "Negative Effects of Acorns on the Wood Mouse Apodemus speciosus," Researches on 
Population Ecology, Vol. 45, No. 1, 2003, pp. 7-17.

[19] A. Carta, "On the Pseudomelanosis of Lymph Nodes in the Acorn-Fed Pigs," SocietàItaliana delle Scienze Veterinarie, Vol. 2, 1948, pp. 363-369.

[20] G. Lanteri, F. Marino, G. Laganà, E. Bellocco, D. Barreca, L. Liotta, A. Sfacteria and B. Macrì, "Acquired Melanosis Caused by Acorn Ingestion in the Nero Siciliano Pig," Veterinary Pathology, Vol. 46, No. 2, 2009, pp. 329-333.

[21] H. O. Gutzeit, Y. Henker, B. Kind and A. Franz, "Specific Interactions of Quercetin and Other Flavonoids with Target Proteins Are Revealed by Elicited Fluorescence," Biochemical and Biophysical Research Communications, Vol. 318, No. 2, 2004, pp. 490-495. doi:10.1016/j.bbrc.2004.04.078

[22] K. Golling, "Polyphenolic Contents of Acorns (Fruits of Quercus spp) and of the Urine of Pigs after the Employment of Acorns as Feed," Thesis Dissertation, University of Veterinary Medicine, Hannover Foundation, 2008, pp. 102-135.

[23] C. Naumann and R. Bassler, "Chemical Analyses of Animal Feed," VDLUFA-Verlag, Darmstadt, 2004.

[24] P. G. Waterman and S. Mole, "Analysis of Plant Metabolites," In: P. G. Waterman and S. Mole, Eds., Methods in Ecology Series, Blackwell Scientific Publications, Oxford, 1994.

[25] P. S. Marcato, "Swine Pathology-Textbook and Atlas," Agricole, Bologna, 1998.

[26] W. R. Zipfel, R. M. Williams, R. Christie, A. Y. Nikitin, B. T. Hyman and W. W. Webb, "Live Tissue Intrinsic Emission Microscopy Using Multiphoton-Excited Native Fluorescence and Second Harmonic Generation," PNAS, Vol. 100, No. 12, 2003, pp. 7075-7080. doi: $10.1073 /$ pnas. 0832308100

ME: Metabolizable Energy

Trp: Triptofan

TPR: True Positive Rate

TNR: True Negative Rate

Acc: Accuracy

NADH: Nicotinamide adenine dinucleotide

NAD(P)H: Nicotinamide adenine dinucleotide-phosphate 